\title{
One-Way Communication Complexity of Symmetric Boolean Functions ${ }^{1}$
}

\author{
Jan Arpe $^{2} \quad$ Andreas Jakoby $^{3} \quad$ Maciej Liśkiewicz ${ }^{4}$ \\ Institut für Theoretische Informatik, \\ Universität zu Lübeck, Wallstr. 40, 23560 Lübeck, Germany \\ arpe/jakoby/liskiewi@tcs.mu-luebeck.de
}

November 21, 2003

\begin{abstract}
We study deterministic one-way communication complexity of functions with Hankel communication matrices. Some structural properties of such matrices are established and applied to the one-way two-party communication complexity of symmetric Boolean functions. It is shown that the number of required communication bits does not depend on the communication direction, provided that neither direction needs maximum complexity. Moreover, in order to obtain an optimal protocol, it is in any case sufficient to consider only the communication direction from the party with the shorter input to the other party. These facts do not hold for arbitrary Boolean functions in general. Next, gaps between one-way and twoway communication complexity for symmetric Boolean functions are discussed. Finally, we give some generalizations to the case of multiple parties.
\end{abstract}

\section{Introduction}

The communication complexity of two-party protocols was introduced by Yao [16] in 1979. The theory of communication complexity evolved into an important branch of computational complexity (for a general survey of the theory see e.g. Kushilevitz and Nisan [9]).

\footnotetext{
${ }^{1}$ A preliminary version of these results has been presented at 14. FCT 2003, Springer, LNCS 2751.

${ }^{2}$ Supported by DFG research grant Re 672/3.

${ }^{3}$ Part of this work was done while visiting International University Bremen, Germany.

${ }^{4}$ On leave from Instytut Informatyki, Uniwersytet Wrocławski, Wrocław, Poland.
} 
In this paper we consider one-way communication, i.e. we restrict the communication to a single round. This simple model has been investigated by several authors for different types of communication such as fully deterministic, probabilistic, nondeterministic, and quantum (see e.g. $[16,12,1,11,3,8,7]$ ). We study the deterministic setting. One-way communication complexity finds application in a wide range of areas, e.g. it provides lower bounds on VLSI complexity and on the size of finite automata (cf. [5]). Moreover, one-way communication complexity of symmetric Boolean functions is connected with binary decision diagrams by the following observation due to Wegener [15] (see also [13]): The size of an optimal protocol coincides with the number of nodes at a certain level in a minimal OBDD.

We consider the standard two-party communication model: Initially the parties, called Alice and Bob, hold disjoint parts of input data $x$ and $y$, respectively. In order to compute a function $f(x, y)$, they exchange messages between each other according to a communication protocol.

In a (deterministic) one-way protocol $\mathcal{P}$ for $f$, one of the parties sends a single message to the other party, and then the latter party computes the output $f(x, y)$. We call $\mathcal{P}$ a protocol of type $A \rightarrow B$ if Alice sends to Bob and of type $B \rightarrow A$ if Bob sends to Alice. The size of $\mathcal{P}$ is the number of different messages that can potentially be transmitted via the communication channel according to $\mathcal{P}$. The one-way communication size $S^{A \rightarrow B}(f)$ of $f$ is the size of the best protocol of type $A \rightarrow B$. It is clear that the respective one-way communication complexity is $C^{A \rightarrow B}(f)=\left\lceil\log S^{A \rightarrow B}(f)\right\rceil$. For the case when Bob sends messages to Alice, we analogously use the notation $S^{B \rightarrow A}$ and $C^{B \rightarrow A}$. Note that throughout this paper, log always denotes the binary logarithm.

The main results of this paper deal with one-way communication complexity of symmetric Boolean functions - an important subclass of all Boolean functions. A Boolean function $F$ is called symmetric, if permuting the input bits does not affect the function value. Some examples for symmetric functions are and, or, parity, majority, and arbitrary threshold functions. We assume that to compute $F$ Alice holds $m$ input bits and Bob holds $n$ bits. As the function value of a symmetric Boolean function only depends on the number of 1's in the input (cf. [14]), it is completely determined by the sum of the number of 1's in Alice's input part and the number of 1's in Bob's part. Hence for such functions, we are faced with the problem of determining the one-way communication complexity of a function

$$
f:\{0, \ldots, m\} \times\{0, \ldots, n\} \rightarrow\{0,1\}
$$

associated to $F$, where $f(x, y)$ only depends on the sum $x+y$. Note that $S^{A \rightarrow B}(F) \leq$ $m+1$ is a trivial upper bound on the one-way communication size of $F$.

Let us assume that Alice's input part is at most as large as Bob's is (i.e. let $m \leq$ $n$ ). While for arbitrary functions this property does not imply which communication direction admits the better one-way protocols, we show that the converse is true for symmetric Boolean functions $F$, namely in this case we have $C^{A \rightarrow B}(F) \leq C^{B \rightarrow A}(F)$. Moreover, we prove that if some protocol of type $A \rightarrow B$ does not require maximal 
size, i.e. if $S^{A \rightarrow B}(F)<m+1$, then both directions yield the same complexities, i.e. $C^{A \rightarrow B}(F)=C^{B \rightarrow A}(F)$.

We also present a class of families of symmetric Boolean functions for which oneway communication is almost as powerful as two-way communication. More precisely, for any family of symmetric Boolean functions $F_{1}, F_{2}, F_{3} \ldots$ with

$$
F_{m}:\{0,1\}^{2 m} \rightarrow\{0,1\},
$$

let $f_{m}:\{0, \ldots, m\} \times\{0, \ldots, m\} \rightarrow\{0,1\}$ denote the integer function associated to $F_{m}$. We prove that if $f_{m} \subseteq f_{m+1}$ for all $m \in \mathbb{N}$, then either the one-way communication complexities of $F_{1}, F_{2}, F_{3} \ldots$ are almost all equal to a constant $c$ or the two-way communication complexities of $F_{1}, F_{2}, F_{3} \ldots$ are infinitely often maximal. We show that one can easily test whether the first or the second case occurs: The two-way communication complexities are infinitely often maximal if and only if the unary language $\left\{0^{k+\ell} \mid f_{k+\ell}(k, \ell)=1, k, \ell \in \mathbb{N}\right\}$ is nonregular.

On the other hand, we construct an example of a symmetric Boolean function having one-way communication complexity exponentially larger than its two-way communication complexity. Finally, we generalize the two-party model to the case of multiple parties and extend our results to such a setting.

Our proofs are based on the fact that the communication matrix of the integer function $f$ associated with a symmetric Boolean function $F$ is a Hankel matrix. In general, the entries of the communication matrix $M_{f}$ of $f$ are defined by $m_{i, j}=f(i, j)$. A Hankel matrix is a matrix in which the entries on each anti-diagonal are constant (equivalently, $m_{i, j}$ only depends on $i+j$ ). Hankel matrices are completely determined by the entries of their first rows and their last columns. Thus with any $(m+1) \times(n+$ 1)-Hankel matrix $H$ we associate a function $f_{H}$ such that $f_{H}(0), f_{H}(1), \ldots, f_{H}(n)$ compose the first row of $H$ and $f_{H}(n), f_{H}(n+1), \ldots, f_{H}(m+n)$ make up its last column. One of the main technical contributions of this paper is a theorem saying that if $m \leq n$ and $H$ has less than $m+1$ different rows, then $f_{H}$ is periodic on a certain large interval. We apply this property to the one-way communication size using a known relationship between this measure and the number of different rows in communication matrices.

As a byproduct, we obtain a word combinatorial property: Let $w$ be an arbitrary string over some alphabet $\Sigma$. Then, for $m \leq\lceil|w| / 2\rceil$ and $n=|w|-m+1$, the number of different substrings of $w$ of length $n$ is at most as large as the number of different substrings of $w$ of length $m$. Moreover, if the former number is strictly less than $m$ (note that it can be at most $m$ in general), then the number of different substrings of length $n$ and the number of different substrings of length $m$ coincide.

The paper is organized as follows: In Section 2, we introduce basic definitions and notation. Section 3 deals with the examination of the number of different rows and columns in Hankel matrices involving certain periodicity properties. In Section 4, we state some applications of these properties. Then, in Section 5, we present a class of symmetric Boolean functions with both maximal one-way and two-way communication complexity, and then we construct a symmetric Boolean function with an 
exponential gap between its one-way and its two-way communication complexity. Finally, in Section 6, we discuss natural extensions of our results to the case of multiple parties.

\section{Preliminaries}

For any integers $0 \leq k<k^{\prime}$, let $\left[k . . k^{\prime}\right]$ denote the set $\left\{k, k+1, \ldots, k^{\prime}\right\}$, and denote $[0 . . k]$ by $[k]$ for short. By $\mathbb{N}$ we denote the set of nonnegative integers. We consider deterministic one-way communication protocols between Alice and Bob for functions $f:[m] \times[n] \rightarrow \Sigma$, where $\Sigma$ is an arbitrary (finite or infinite) nonempty set. More specifically, we assume that Alice holds a value $x \in[m]$, and Bob holds a value $y \in[n]$ for some fixed positive integers $m$ and $n$. Their aim is to compute the value $f(x, y)$.

Let $\mathcal{M}(m, n)$ denote the set of all $(m+1) \times(n+1)$ matrices $M=\left(m_{i, j}\right)$ with $m_{i, j} \in \Sigma$. It will be convenient for us to enumerate the rows from 0 to $m$ and the columns from 0 to $n$. For a given function $f:[m] \times[n] \rightarrow \Sigma$, we denote by $M_{f}$ the corresponding communication matrix in $\mathcal{M}(m, n)$.

Definition 1 For a matrix $M \in \mathcal{M}(m, n)$, define \#row $(M)$ to be the number of different rows of $M$, and similarly let $\# \operatorname{col}(M)$ be the number of different columns of $M$. Furthermore, for any $i, j \in[\mathrm{m}]$, let $i \sim_{M} j$ denote that the rows $i$ and $j$ of $M$ are equal.

Since the sender has to specify the type of row (resp. column) his input belongs to, it is easy to characterize the one-way communication size by \#row and \#col.

Fact 1 For all $m, n \in \mathbb{N}$ and for every function $f:[m] \times[n] \rightarrow \Sigma$, it holds that $S^{A \rightarrow B}(f)=\# \operatorname{row}\left(M_{f}\right)$ and $S^{B \rightarrow A}(f)=\# \operatorname{col}\left(M_{f}\right)$.

In this paper we will restrict ourselves to functions $f$ that only depend on the sum of the arguments. Note that for such functions $f$ the communication matrix $M_{f}$ is a Hankel matrix. The problem of finding protocols for such restricted $f$ arises naturally when one considers symmetric Boolean functions.

Definition 2 Let $f:[s] \rightarrow \mathbb{N}, \lambda \geq 1$ and $s_{1}, s_{2} \in[s]$ with $s_{1} \leq s_{2}-\lambda$. We call $f$ $\lambda$-periodic on $\left[s_{1} . . s_{2}\right]$, if for all $x \in\left[s_{1} . . s_{2}-\lambda\right], f(x)=f(x+\lambda)$.

Obviously, $f$ is $\lambda$-periodic on $\left[s_{1} . . s_{2}\right]$ if and only if for all $x, x^{\prime} \in\left[s_{1} . . s_{2}\right]$ with $\lambda \mid$ $\left(x-x^{\prime}\right)$, it holds that $f(x)=f\left(x^{\prime}\right)$.

\section{Periodicity of Rows and Columns in Hankel Matrices}

This section is devoted to examine the relationship between the number of different rows and the number of different columns in a Hankel matrix. Lemmas 1 through 3 
are technical preparations for Theorem 1 which gives an explicit characterization of a certain periodic behaviour of the function associated with a Hankel matrix and of the Hankel matrix itself. Theorems 2 and 3 reveal all possible constellations of values for \#row $(H)$ and \#col $(H)$ for a Hankel matrix $H$. The results will be applied to the theory of one-way communication in Section 4.

Fact 2 Let $f:[s] \rightarrow \mathbb{N}$ be $\lambda$-periodic on $\left[s_{1} . . s_{2}\right] \subseteq[s]$ and on $\left[t_{1} . . t_{2}\right] \subseteq[s]$ such that $s_{1} \leq t_{1}$ and $t_{1}+\lambda \leq s_{2}$. Then $f$ is $\lambda$-periodic on $\left[s_{1}\right.$..t $\left.t_{2}\right]$.

Proof: Let $x \in\left[s_{1} . . t_{2}-\lambda\right]$. If $x \leq t_{1}$, then $s_{1} \leq x \leq x+\lambda \leq t_{1}+\lambda \leq s_{2}$, so $f(x)=f(x+\lambda)$ because of the $\lambda$-periodicity on $\left[s_{1} . . s_{2}\right]$. On the other hand, if $x>t_{1}$, then $f(x)=f(x+\lambda)$ because of the $\lambda$-periodicity on $\left[t_{1} . . t_{2}\right]$.

Lemma 1 Let $H \in \mathcal{M}(m, n)$ be a Hankel matrix, $m_{0}, m_{1} \in[m]$ with $m_{0}<m_{1}$, and $\lambda \in\left[1 . . m_{1}-m_{0}\right]$. Then the following two statements are equivalent:

(a) $f_{H}$ is $\lambda$-periodic on $\left[m_{0} . . m_{1}+n\right]$.

(b) For all $x \in\left[m_{0} . . m_{1}\right]$ and all $k \in \mathbb{N}$ such that $x+k \lambda \leq m_{1}, x \sim_{H} x+k \lambda$.

Proof: “(a) $\Rightarrow$ (b)": Let $x \in\left[m_{0} . . m_{1}\right]$ and $k \in \mathbb{N}$ such that $x+k \lambda \leq m_{1}$. For all $y \in[n]$,

$$
x+y \geq m_{0} \quad \text { and } \quad x+y+k \lambda \leq m_{1}+n .
$$

Since $f_{H}$ is $\lambda$-periodic on $\left[m_{0} . . m_{1}+n\right]$, we have $f_{H}(x+y)=f_{H}(x+k \lambda+y)$. "(b) $\Rightarrow$ (a)": Let $x \in\left[m_{0} . . m_{1}+n-\lambda\right]$. We consider two cases. If $x \leq m_{0}+n$, then $f_{H}(x)=f_{H}\left(m_{0}+\left(x-m_{0}\right)\right)=f_{H}\left(m_{0}+\lambda+\left(x-m_{0}\right)\right)=f_{H}(x+\lambda)$, because $m_{0} \sim_{H} m_{0}+\lambda$ by hypothesis. If on the other hand $x>m_{0}+n$, then $x-n>m_{0}$ and $x-n+\lambda \leq m_{1}$. By hypothesis, $x-n \sim_{H} x-n+\lambda$, and thus $f_{H}(x)=f_{H}(x-n+n)=f_{H}(x-n+\lambda+n)=f_{H}(x+\lambda)$.

Corollary 1 Let $H \in \mathcal{M}(m, n)$ be a Hankel matrix and $i, j \in[m]$ with $i<j$. Then $i \sim_{H} j$ if and only if $f_{H}$ is $(j-i)$-periodic on $[i . . j+n]$.

Corollary 2 Let $H \in \mathcal{M}(m, n)$ be a Hankel matrix. If $f_{H}$ is $\lambda$-periodic on $\left[m_{0} . . m_{1}+\right.$ $n]$ for some $m_{0}, m_{1} \in[m]$ with $m_{0}<m_{1}$ and some $\lambda \in\left[1 . . m_{1}-m_{0}\right]$, then \#row $(H) \leq$ $m_{0}+\lambda+m-m_{1}$, where equality holds if and only if all rows $0, \ldots, m_{0}+\lambda-1$ and $m_{1}+1, \ldots, m$ are pairwise different.

Lemma 2 Let $H \in \mathcal{M}(m, n)$ be a Hankel matrix and $m_{0}, m_{0}^{\prime}, i, j \in[m]$ such that $m_{0} \leq i<j, m_{0}^{\prime}-m_{0} \leq n+1, j-m_{0} \leq n+1, i \sim_{H} j$, and $m_{0} \sim_{H} m_{0}^{\prime}$. Then $f_{H} i s$ $(j-i)$-periodic on $\left[m_{0} . . j+n\right]$.

Proof: Choose $\lambda=j-i$ and $\mu_{0}=m_{0}^{\prime}-m_{0}$. By Corollary $1, f_{H}$ is

(i) $\mu_{0}$-periodic on $\left[m_{0} . . m_{0}^{\prime}+n\right]$ and 


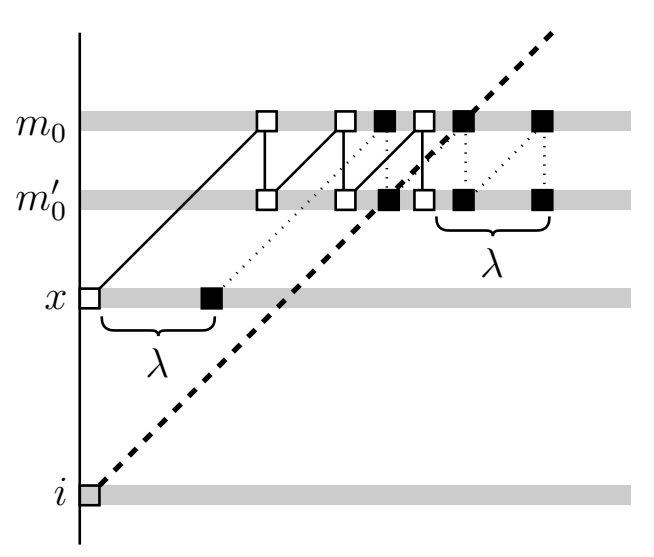

Figure 1: An illustration of Case 1.

(ii) $\lambda$-periodic on $[i . . j+n]$.

Let $x \in\left[m_{0} . . j+n-\lambda\right]$. In order to show that $f_{H}(x+\lambda)=f_{H}(x)$, we consider:

Case 1: $m_{0} \leq x<i$ : Let $k \in \mathbb{N}$ such that $i \leq x+k \mu_{0} \leq i+\mu_{0}-1$. We need to show that

$$
\begin{aligned}
x, x+k \mu_{0}, x+k \mu_{0}+\lambda, x+\lambda & \in\left[m_{0} . . m_{0}^{\prime}+n\right] \text { and } \\
x+k \mu_{0}, x+k \mu_{0}+\lambda & \in[i . . j+n]
\end{aligned}
$$

in order to apply properties (i) and (ii) to the corresponding elements. Property (1) follows from $m_{0} \leq x$ and $x+k \mu_{0}+\lambda \leq i+\mu_{0}+\lambda-1=j+m_{0}^{\prime}-m_{0}-1 \leq m_{0}^{\prime}+n$. Property (2) is due to $i \leq x+k \mu_{0}$ and $x+k \mu_{0}+\lambda \leq j-1+\mu_{0} \leq j+n$. Now (cf. Fig. 1) $f_{H}(x)=f_{H}\left(x+k \mu_{0}\right)=f_{H}\left(x+k \mu_{0}+\lambda\right)=f_{H}(x+\lambda)$, where the first and the last equality follow from properties (1) and (i), and the middle equality is due to properties (2) and (ii).

Case 2: $i \leq x \leq j+n-\lambda$ : In this case, $f_{H}(x)=f_{H}(x+\lambda)$ by Corollary 1 .

The following Lemma is symmetric to the previous one:

Lemma 3 Let $H \in \mathcal{M}(m, n)$ be a Hankel matrix and $m_{1}, m_{1}^{\prime}, i, j \in[m]$ such that $i<j \leq m_{1}, m_{1}-m_{1}^{\prime} \leq n+1, m_{1}-i \leq n+1, i \sim_{H} j$, and $m_{1} \sim_{H} m_{1}^{\prime}$. Then $f_{H} i s$ $(j-i)$-periodic on $\left[i . m_{1}+n\right]$.

Proof: $\quad$ Let $H=\left(h_{i, j}\right)$. We define $\lambda=j-i$ and $H^{\prime}=\left(h_{\mu, \nu}^{\prime}\right) \in \mathcal{M}(m, n)$ by $h_{\mu, \nu}^{\prime}=h_{m-\mu, n-\nu}$ for $(\mu, \nu) \in[m] \times[n]$, i.e. we rotate $H$ by 180 degrees in the plane. Clearly, $H^{\prime}$ is again a Hankel matrix. Moreover, we have $f_{H}(z)=f_{H^{\prime}}(m+n-z)$ for all $z \in[m+n]$. We set $m_{0}=m-m_{1}, m_{0}^{\prime}=m-m_{1}^{\prime}, i^{\prime}=m-j$, and $j^{\prime}=m-i$. 
Now it is easy to check that $H^{\prime}, i^{\prime}, j^{\prime}, m_{0}$, and $m_{0}^{\prime}$ fulfill the preconditions of Lemma 2 and $m+n-x-\lambda \in\left[m_{0} . . j^{\prime}+n-\lambda\right]$, thus yielding

$$
f_{H}(x+\lambda)=f_{H^{\prime}}(m+n-x-\lambda)=f_{H^{\prime}}(m+n-x)=f_{H}(x) .
$$

Theorem 1 Let $m \leq n+1$ and $H \in \mathcal{M}(m, n)$ be a Hankel matrix with \#row $(H)<$ $m+1$. Then there exist $\lambda \in[1 . . n]$ and $m_{0}, m_{1} \in[m]$ with $m_{1}-m_{0} \geq \lambda$ such that the following two properties hold:

(a) The function $f_{H}$ is $\lambda$-periodic on $\left[m_{0} . . m_{1}+n\right]$.

(b) If $i, j \in[m]$ with $i<j$ and $i \sim_{H} j$, then $i, j \in\left[m_{0} . . m_{1}\right]$ and $\lambda \mid(j-i)$.

Moreover, $m_{0}, m_{1}$ and $\lambda$ can be explicitly determined as follows:

$$
\begin{aligned}
m_{0} & =\min \left\{k \in[m] \mid \exists k^{\prime} \in[m] \text { with } k^{\prime}>k \text { and } k \sim_{H} k^{\prime}\right\}, \\
m_{1} & =\max \left\{k \in[m] \mid \exists k^{\prime} \in[m] \text { with } k^{\prime}<k \text { and } k \sim_{H} k^{\prime}\right\}, \text { and } \\
\lambda & =\min \left\{j-i \mid i, j \in[m] \text { with } i \sim_{H} j \text { and } i<j\right\} .
\end{aligned}
$$

Proof: Since \#row $(H)<m+1$, there exist $i, j \in[m]$ with $i<j$ such that $i \sim_{H} j$. Thus, $m_{0}, m_{1}$ and $\lambda$ are well-defined. Clearly, $m_{1}-m_{0} \geq \lambda$. Choose $i_{0}, j_{0} \in[m]$ such that $i_{0} \sim_{H} j_{0}$ and $j_{0}-i_{0}=\lambda$. Since $m \leq n$, all preconditions of Lemma 2 and Lemma 3 are satisfied. Thus we conclude that $f_{H}$ is $\lambda$-periodic on both discrete intervals $\left[m_{0} . . j_{0}+n\right]$ and $\left[i_{0} . . m_{1}+n\right]$. Fact 2 now yields property (a). Now let $i, j \in[m]$ with $i<j$ and $i \sim_{H} j$. Let $k \in \mathbb{N}$ such that $j-i=k \lambda+r$ with $0 \leq r<\lambda$. By property (a), $f_{H}$ is $\lambda$-periodic on $\left[m_{0} . . m_{1}+n\right]$, and so by Lemma 1 (note that $i+k \lambda=j-r \leq j \leq m_{1}$ ), we have $i+k \lambda \sim_{H} i \sim_{H} j$. As $r=j-i-k \lambda<\lambda$ and $\lambda$ is the minimal difference between two equal rows of different indices, we have $r=0$, so $\lambda \mid(j-i)$.

Using Corollary 2 we deduce two consequences of Theorem 1:

Corollary 3 For $H, m_{0}, m_{1}$ and $\lambda$ as in Theorem 1 , \#row $(H)=m_{0}+\lambda+m-m_{1}$, i.e. $H$ has exactly $m_{0}+\lambda+m-m_{1}$ pairwise different rows.

Corollary 4 Let $m \leq n+1$ and $H \in \mathcal{M}(m, n)$ be a Hankel matrix with \#row $(H)<$ $m+1$. Then $\# \operatorname{col}(H) \leq \# \operatorname{row}(H)$.

Proof: Let $m_{0}, m_{1}$ and $\lambda$ be as in Theorem 1. From Theorem 1, we have that the function $f_{H}=f_{H^{T}}$ is $\lambda$-periodic on $\left[m_{0} . . m_{1}+n\right]=\left[m_{0} . .\left(m_{1}+n-m\right)+m\right]$. Now Corollary 2 implies that

$$
\text { \#row }\left(H^{T}\right) \leq m_{0}+\lambda+n-\left(m_{1}+n-m\right)=m_{0}+\lambda+m-m_{1}=\text { \#row }(H),
$$

where the last equality is due to Corollary 3 . Hence the corollary follows since we have $\# \operatorname{col}(H)=\# \operatorname{row}\left(H^{T}\right)$.

The next lemma states an "expansion property" of Hankel matrices with at least two equal rows. 
Lemma 4 For arbitrary $m, n \in \mathbb{N}$ let $H \in \mathcal{M}(m, n)$ be a Hankel matrix with \#row $(H)<m+1$. Then there exist $m^{\prime} \geq n$ and a Hankel matrix $\tilde{H} \in \mathcal{M}\left(m^{\prime}, n\right)$ such that $\# \operatorname{row}(\tilde{H})=\# \operatorname{row}(H)$ and $\# \operatorname{col}(\tilde{H})=\# \operatorname{col}(H)$.

Proof: We duplicate the area between two equal rows until the total number of rows exceeds the total number of columns $n$. This process effects neither the number of different rows nor the number of different columns. To do this we proceed as follows. Since \#row $(H)<m+1$, there exist $m_{0}, m_{1} \in[m]$ with $m_{0}<m_{1}$ and $m_{0} \sim_{H} m_{1}$. Set $\lambda=m_{1}-m_{0}$, and let $c \in \mathbb{N}$ such that $m+c \lambda \geq n$. We set $m^{\prime}=m+c \lambda$ and define $\tilde{H}=\left(\tilde{h}_{i, j}\right) \in \mathcal{M}\left(m^{\prime}, n\right)$, where for $j \in[n]$,

$$
\tilde{h}_{i, j}= \begin{cases}h_{i, j} & \text { if } i<m_{0}, \\ h_{m_{0}+\ell, j} & \text { if } i=m_{0}+k \lambda+\ell \text { for some } k \in[c] \text { and some } \ell \in[\lambda-1], \\ h_{i-c \lambda, j}, & \text { if } i \geq m_{1}+c \lambda .\end{cases}
$$

Now, $\tilde{H}$ is again a Hankel matrix, and both properties \# $\operatorname{row}(\tilde{H})=\# \operatorname{row}(H)$ and $\# \operatorname{col}(\tilde{H})=\# \operatorname{col}(H)$ hold.

Theorem 2 Let $m \leq n+1$ and $H \in \mathcal{M}(m, n)$ be a Hankel matrix with \#row $(H)<$ $m+1$. Then \#row $(H)=\# \operatorname{col}(H)$.

Proof: From Corollary 4, we have \#row $(H) \geq \# \operatorname{col}(H)$. By Lemma 4, there exist $m^{\prime} \geq n$ and a Hankel matrix $\tilde{H} \in \mathcal{M}\left(m^{\prime}, n\right)$ such that \#row $(\tilde{H})=$ \#row $(H)$ and $\# \operatorname{col}(\tilde{H})=\# \operatorname{col}(H)$. Thus, again by Corollary 4, we obtain \#row $(H)=\# \operatorname{row}(\tilde{H})=$ $\# \operatorname{col}\left(\tilde{H}^{T}\right) \leq \# \operatorname{row}\left(\tilde{H}^{T}\right)=\# \operatorname{col}(\tilde{H})=\# \operatorname{col}(H)$. Consequently, we have \#row $(H)=$ $\# \operatorname{col}(H)$.

Theorem 3 Let $m \leq n$ and $H \in \mathcal{M}(m, n)$ be a Hankel matrix with \#row $(H)=$ $m+1$. Then $\# \operatorname{col}(H) \geq m+1$.

Proof: Induction on $n$ : For $n=m$, we have $H=H^{T}$ and thus

$$
\# \operatorname{col}(H)=\# \operatorname{row}\left(H^{T}\right)=\# \operatorname{row}(H)=m+1 .
$$

Now suppose that $n>m$. Let $H^{\prime} \in \mathcal{M}(m, n-1)$ be the matrix $H$ without its last column. We consider two cases:

Case 1: $n \sim_{H^{T}} n^{\prime}$ for some $n^{\prime} \in[n-1]$. Then $\# \operatorname{col}(H)=\# \operatorname{col}\left(H^{\prime}\right)$. In addition, \#row $\left(H^{\prime}\right)=m+1$, because if \#row $\left(H^{\prime}\right) \leq m$ was true, then we had $i \sim_{H^{\prime}} j$ for some $0 \leq i<j \leq m$, and thus $i \sim_{H} j$, since $f_{H}(i+n)=f_{H}\left(i+n^{\prime}\right)=f_{H}\left(j+n^{\prime}\right)=$ $f_{H}(j+n)$. Thus, we get $\# \operatorname{col}(H)=\# \operatorname{col}\left(H^{\prime}\right) \geq m+1$ by induction hypothesis.

Case 2: $n \chi_{H^{T}} n^{\prime}$ for all $n^{\prime} \in[n-1]$. Then $\# \operatorname{col}(H)=\# \operatorname{col}\left(H^{\prime}\right)+1$. Once again, we have to consider two subcases:

Case 2a: $\# \operatorname{row}\left(H^{\prime}\right)=m+1$ : Then $\# \operatorname{col}(H)=\# \operatorname{col}\left(H^{\prime}\right)+1=m+2>m+1$ by 
induction hypothesis.

Case 2b: \#row $\left(H^{\prime}\right) \leq m$ : Assume that \#row $\left(H^{\prime}\right)<m$, and let

$$
\begin{aligned}
m_{0} & =\min \left\{k \in[m] \mid \exists k^{\prime} \in[m] \text { with } k^{\prime}>k \text { and } k \sim_{H} k^{\prime}\right\}, \\
m_{1} & =\max \left\{k \in[m] \mid \exists k^{\prime} \in[m] \text { with } k^{\prime}<k \text { and } k \sim_{H} k^{\prime}\right\}, \\
\lambda & =\min \left\{k^{\prime}-k \mid k, k^{\prime} \in[m] \text { with } k<k^{\prime} \text { and } k \sim_{H} k^{\prime}\right\},
\end{aligned}
$$

where $m_{0}^{\prime}, m_{1}^{\prime}$ and $\lambda^{\prime}$ are the corresponding numbers for $H^{\prime}$. By Corollary 3 , we have \#row $\left(H^{\prime}\right)=m_{0}^{\prime}+m-m_{1}^{\prime}+\lambda^{\prime}$, and by Theorem $1 f$ is $\lambda^{\prime}$-periodic on $\left[m_{0}^{\prime} . . m_{1}^{\prime}+n-1\right]$. Since \#row $\left(H^{\prime}\right)<m$ by assumption, $\lambda^{\prime}<m_{1}^{\prime}-m_{0}^{\prime}$. In particular, $m_{0} \sim_{H} m_{0}+\lambda^{\prime}$, and thus $\lambda \mid \lambda^{\prime}$ by Theorem 1. Consequently, $m_{0} \leq m_{0}^{\prime}, m_{1} \geq m_{1}^{\prime}-1$ and $\lambda \leq \lambda^{\prime}$. Hence again by Corollary 3 ,

$$
\begin{aligned}
\# \operatorname{row}(H) & =m_{0}+m-m_{1}+\lambda \leq m_{0}^{\prime}+m-\left(m_{1}^{\prime}-1\right)+\lambda^{\prime} \\
& \leq m_{0}^{\prime}+m-m_{1}^{\prime}+\lambda^{\prime}+1=\# \operatorname{row}\left(H^{\prime}\right)+1<m+1,
\end{aligned}
$$

contradicting the precondition \#row $(H)=m+1$. Thus, \#row $\left(H^{\prime}\right)=m$. By Theo$\operatorname{rem} 2, \# \operatorname{col}\left(H^{\prime}\right)=\# \operatorname{row}\left(H^{\prime}\right)=m$. Consequently, $\# \operatorname{col}(H)=\# \operatorname{col}\left(H^{\prime}\right)+1=m+1$.

Note that for Hankel matrices over $\Sigma$ with $|\Sigma| \geq m+n+1$ we can say even more. Namely, if $m \leq n$, then for all $r \in[m+1 . . n+1]$, there exists a Hankel matrix $H \in \mathcal{M}(m, n)$ with \#row $(H)=m+1$ and $\# \operatorname{col}(H)=r$. To see this, define $f:[m] \times[n] \rightarrow \Sigma=\left\{a_{0}, \ldots, a_{m+n}\right\}$ by $f(x, y)=a_{(x+y)} \bmod r$. Then $H=M_{f}$ is a Hankel matrix fulfilling the requested properties.

\section{Applications}

Theorems 2 and 3 can be summarized in terms of one-way communication as follows.

Theorem 4 Let $m \leq n$ and $f:[m] \times[n] \rightarrow \Sigma$ be a function for which the corresponding communication matrix $M_{f}$ is a Hankel matrix. Then the following properties hold:

(a) $S^{A \rightarrow B}(f) \leq S^{B \rightarrow A}(f)$.

(b) If $S^{A \rightarrow B}(f)<m+1$, then $S^{A \rightarrow B}(f)=S^{B \rightarrow A}(f)$.

This result can immediately be applied to symmetric Boolean functions:

Corollary 5 Let $m \leq n$ and $F:\{0,1\}^{m} \times\{0,1\}^{n} \rightarrow\{0,1\}$ be a symmetric Boolean function. Then the following properties hold:

(a) $S^{A \rightarrow B}(F) \leq S^{B \rightarrow A}(F)$.

(b) If $S^{A \rightarrow B}(F)<m+1$, then $S^{A \rightarrow B}(F)=S^{B \rightarrow A}(F)$. 
Proof: The communication matrix $M_{f}$ of the function $f:[m] \times[n] \rightarrow\{0,1\}$ defined by

$$
f(x, y)=F((\underbrace{1, \ldots, 1}_{x}, \underbrace{0, \ldots, 0}_{m-x}),(\underbrace{1, \ldots, 1}_{y}, \underbrace{0, \ldots, 0}_{n-y}))
$$

is a Hankel matrix. Thus the claim follows from Theorem 4.

The results of the last paragraph can also be applied to word combinatorics as follows:

Theorem 5 Let $w$ be an arbitrary string over some alphabet $\Sigma$, and let $N_{w}(i)$ denote the number of different subwords of $w$ of length $i$. Then, for $m \leq\lceil|w| / 2\rceil$ and $n=$ $|w|-m+1$, we have $N_{w}(n) \leq N_{w}(m)$. Moreover, if $N_{w}(n)<m$ (note that $N_{w}(n) \leq$ $m$ in general), then $N_{w}(n)=N_{w}(m)$.

Proof: $\quad$ Let $m \leq\lceil|w| / 2\rceil, n=|w|-m+1$, and $w=w_{1} \ldots w_{m+n-1}$ with $w_{i} \in \Sigma$ for $1 \leq i \leq m+n-1$. Define the Hankel matrix $H=\left(h_{i, j}\right) \in \mathcal{M}_{\Sigma}(m-1, n-1)$ by $h_{i, j}=w_{i+j+1}$. The rows of $H$ make up the subwords of $w$ of length $n$, while the columns of $H$ compose the subwords of $w$ of length $m$. Now Theorem 2 and Theorem 3 prove the claim.

\section{One-Way versus Two-Way Protocols}

In this section we first present a class of families of functions for which one-way communication complexties are almost the same as two-way communication complexties. We denote the two-way complexity of $F$ by $C(F)$. Let $F_{1}, F_{2}, F_{3} \ldots$ with $F_{m}:\{0,1\}^{2 m} \rightarrow\{0,1\}$ be a family of symmetric Boolean functions and let $f_{m}:[m] \times$ $[m] \rightarrow\{0,1\}$ denote the integer function associated to $F_{m}$, i.e. $F\left(x_{1}, \ldots, x_{2 m}\right)=1$ if and only if $f\left(\sum_{i=1}^{m} x_{i}, \sum_{i=m+1}^{2 m} x_{i}\right)=1$.

Theorem 6 Let $F_{1}, F_{2}, F_{3} \ldots$ be a family of symmetric Boolean functions such that $f_{m} \subseteq f_{m+1}$ for all $m \in \mathbb{N}$. Then either

(a) for almost all $m \in \mathbb{N}, C^{A \rightarrow B}\left(F_{m}\right)=c$ for some constant $c$ or

(b) for infinitely many $m \in \mathbb{N}, C\left(F_{m}\right)=\lceil\log (m+1)\rceil$.

Moreover, (b) holds iff the language $L=\left\{0^{k+\ell} \mid f_{k+\ell}(k, \ell)=1, k, \ell \in \mathbb{N}\right\}$ is nonregular.

Proof: First, Theorem 11.3 in [6] gives a nice characterization of (non)regular unary languages in terms of the rank of certain Hankel matrices. This characterization was first observed by Condon et al. [2]. It says that the unary language $L$ is nonregular if and only if for infinitely many $m \in \mathbb{N}, \operatorname{rank}\left(M_{f_{m}}\right)=m+1$ (i.e. the communication matrix $M_{f_{m}}$ has maximum rank). Second, Mehlhorn and Schmidt [10] showed that $C(f) \geq \log \left(\operatorname{rank}\left(M_{f}\right)\right)$ for every $f$. Combining these facts we get that for nonregular $L, C\left(f_{m}\right)=\lceil\log (m+1)\rceil$ for infinitely many $m \in \mathbb{N}$. 
On the other hand, if $L$ is regular then by the Myhill-Nerode Theorem [4] the infinite matrix $M=\left(m_{i, j}\right)_{i, j \in \mathbb{N}}$ defined by $m_{i, j}=1$ iff $0^{i+j} \in L$, has constant number of different rows. Hence the theorem follows.

Example 1 Let $F_{m}\left(x_{1}, x_{2}, \ldots, x_{2 m}\right)=1$ if and only if the number of 1 's in the sequence $x_{1}, x_{2}, \ldots, x_{2 m}$ is the square of some integer. By Theorem 6 either for all $m \in \mathbb{N}, C\left(F_{m}\right), C^{A \rightarrow B}\left(F_{m}\right) \leq c$ for some constant $c$ or for infinitely many $m \in \mathbb{N}$, $C^{A \rightarrow B}\left(F_{m}\right)=C\left(F_{m}\right)=\lceil\log (m+1)\rceil$. Since the language $\left\{0^{n} \mid n\right.$ is the square of some integer $\}$ is nonregular, the (one-way) communication complexity of $F_{m}$ is maximal for infinitely many $m \in \mathbb{N}$.

Next, we construct a symmetric Boolean function with an exponential difference between its one-way and its two-way communication complexity. Let $p_{0}, p_{1}, \ldots$ with $p_{i}<p_{i+1}$ for all $i \in \mathbb{N}$ be the sequence of all prime numbers. According to the Prime Number Theorem, there are at least $\frac{\ell}{\log \ell}$ prime numbers in the interval $[\ell]$ for all $\ell \geq 5$. For $k=\lceil\log \log m\rceil$ and $n=2^{k} \cdot\left(1+\prod_{i=0}^{2^{k}-1} p_{i}\right)$, consider the function $f:[m] \times[n] \rightarrow\{0,1\}$ defined by $f(x, y)=1$ iff $\left\lfloor\frac{z}{2^{k}}\right\rfloor \bmod p_{z \bmod 2^{k}}=0$, where $z=x+y$. Using the following two-way protocol, one can see that the two-way communication complexity of $f$ is at most $4 \log \log m$ : In the first round, Bob sends $y_{0}=y \bmod 2^{k}$ to Alice. In the second round, Alice sends $z_{0}=\left(x+y_{0}\right) \bmod 2^{k}$ and $z^{\prime}=\left\lfloor\frac{x+y_{0}}{2^{k}}\right\rfloor \bmod p_{z_{0}}$ to Bob. Finally, Bob computes $f(x, y)$ by checking whether $\left(\left\lfloor\frac{y}{2^{k}}\right\rfloor+z^{\prime}\right) \bmod p_{z_{0}}=0$.

Note that $z_{0}=z \bmod 2^{k}$. The correctness of the protocol can be seen by investigating the addition of integers using a remainder representation.

Lemma $5 C(f) \leq 4 \log \log m$.

For the one-way communication complexity of $f$ we obtain:

Lemma 6 \#row $\left(M_{f}\right)=m+1$, i.e. $C^{A \rightarrow B}(f)=\lceil\log (m+1)\rceil$.

The proofs of the lemmas are straightforward. We conclude the section with the following

Theorem 7 For the symmetric Boolean function $F:\{0,1\}^{m} \times\{0,1\}^{n} \rightarrow\{0,1\}$ associated with $f$, we have $C(F) \in O(\log \log m)$ and $C^{A \rightarrow B}(F) \in \Theta(\log m)$.

\section{Multi-party Communication}

So far we have analyzed the case that a fixed input partition for a function is given. However, sometimes it is also of interest to examine the communication complexity of a fixed function under varying the input partition. A typical question for this scenario is whether we can partition the input in such a way that the communication complexities 
for protocols of type $A \rightarrow B$ and $B \rightarrow A$ coincide. The main tool for these examinations is the diversity $\Delta(f)$ of $f$ which we introduce below. For a function $f:[s] \rightarrow \Sigma$ and $m \in[s]$, define $f_{m}:[m] \times[s-m] \rightarrow \Sigma$ by $f_{m}(x, y)=f(x+y)$ for $x \in[m]$ and $y \in[s-m]$, and let $r_{f}(m)=\#$ row $\left(M_{f_{m}}\right)$. We define $\Delta(f)=\max _{m \in[s]} r_{f}(m)$.

Lemma 7 For every function $f:[s] \rightarrow \Sigma$, the following conditions hold:

(a) $r_{f}(m)=m+1$ for all $m \in[\Delta(f)-1]$,

(b) if $\Delta(f) \leq \frac{s}{2}$, then $r_{f}(m)=\Delta(f)$ for all $m \in[\Delta(f)-1 \ldots s-\Delta(f)+1]$,

(c) $r_{f}(m) \geq r_{f}(m+1)$ for all $m \in[\Delta(f)-1 . . s-1]$.

Proof: Obviously, we have $r_{f}(m) \leq m+1$. From the definition of $f_{m}$ we can derive that the communication matrix of $f_{m}$ is a Hankel matrix. The first and last part of the claim follow directly from the following observation:

Assume that for some $m$ we have $r_{f}(m)<m+1$. Then for every $i \geq m$ it holds $r_{f}(i) \geq r_{f}(i+1)$.

Below we show that the observation is true. Note first that if rows $j$ and $k$ in $M_{f_{i}}$ are equal then in $M_{f_{i+1}}$ rows $j$ and $k$ are equal, too. Hence $r_{f}(m)<m+1$ implies that for every $i \geq m$ it holds $r_{f}(i)<i+1$.

Let $k$ be the maximum index of a row in $M_{f_{i}}$ such that for some $j<k$ the rows $j$ and $k$ coincide. Such a pair exists because $r_{f}(i)<i+1$. Since each row $\ell+1$ of $M_{f_{i+1}}$ can be derived from row $\ell$ in $M_{f_{i}}$ by deleting its first entry, it is true that in $M_{f_{i+1}}$ the rows $j+1$ and $k+1$ coincide. If $k=i$, then the number of different rows among the rows $0, \ldots, i$ in $M_{f_{i+1}}$ is at most $r_{f}(i)$, the number of different rows in $M_{f_{i}}$. Since the last row in $M_{f_{i+1}}$ coincides with row $j+1$, we have $r_{f}(i+1) \leq r_{f}(i)$. If on the other hand $k<i$, then the rows $j+1$ and $k+1$ do not coincide in $M_{f_{i}}$ (by maximality of $k$ ), so the number of different rows among rows $1, \ldots, i$ in $M_{f_{i+1}}$ is strictly smaller than in $M_{f_{i}}$. This implies $r_{f}(i+1) \leq\left(r_{f}(i)-1\right)+1=r_{f}(i)$.

Let us now focus on the second part of the claim. Let $m=\Delta(f)-1$ and $n=$ $s-\Delta(f)+1$. Then $m \leq n$ (since $\Delta(f) \leq \frac{s}{2}$ ) and \#row $\left(M_{f_{m}}\right)=\Delta(f)=m+1$ by part (a). From Theorem 3 it follows that $\# \operatorname{col}\left(M_{f_{m}}\right) \geq m+1=\Delta(f)$. Since $M_{f_{s-m}}$ (for arbitrary $m \in[s]$ ) is the transpose of $M_{f_{m}}$, we have $\# \operatorname{col}\left(M_{f_{s-m}}\right)=\# \operatorname{row}\left(M_{f_{s}}\right)$. Consequently,

$$
\begin{aligned}
r_{f}(\Delta(f)-1) & =\Delta(f) \\
& \leq \# \operatorname{col}\left(M_{f_{\Delta(f)-1}}\right) \\
& =\# \operatorname{row}\left(M_{f_{s-\Delta(f)+1}}\right)=r_{f}(s-\Delta(f)+1) .
\end{aligned}
$$

On the other hand, $r_{f}$ is nonincreasing on $[\Delta(f)-1 . . s-1]$ by part (c), so $r_{f}(m)=$ $\Delta(f)$ for all $m \in[\Delta(f)-1 . . s-\Delta(f)+1]$. 
It is an immediate consequence of Lemma 7 that $\Delta(f)$ equals the minimum $m$ such that $M_{f_{m}}$ has less than $m+1$ different rows, provided that such an $m$ exists.

The diversity is helpful to analyze the case that more than two parties are involved. For such multi-party communication we assume that the input is distributed among $d$ parties $P_{1}, \ldots, P_{d}$. Every party $P_{i}$ knows a value $x_{i} \in\left[m_{i}\right]$. The goal is to compute a fixed function $f:\left[m_{1}\right] \times \ldots \times\left[m_{d}\right] \rightarrow \Sigma$. Analogously to communication matrices in the two-party case, we use multidimensional arrays to represent $f$.

Let $\mathcal{M}\left(m_{1}, \ldots, m_{d}\right)$ be the set of all $d$-dimensional $\left(m_{1}+1\right) \times \ldots \times\left(m_{d}+1\right)$ arrays $M$ with entries $M\left(i_{1}, \ldots, i_{d}\right) \in \Sigma$ for $i_{j} \in\left[m_{j}\right], j \in[1 . . d] . M$ is called the communication array of a function $f$ iff $M\left(i_{1}, \ldots, i_{d}\right)=f\left(i_{1}, \ldots, i_{d}\right)$. We denote the communication array of $f$ by $M_{f}$.

Recall that in the two-party model the sender has to specify the type of row/column his input belongs to. In the multi-party case each party has to specify the type of subarray determined by his input value. Therefore, for each $k \in[1 . . d]$ and each $x \in\left[m_{k}\right]$, we define the subarray $M_{x}^{(k)} \in \mathcal{M}\left(m_{1}, \ldots, m_{k-1}, m_{k+1}, \ldots, m_{d}\right)$ of $M$ by $M_{x}^{(k)}\left(i_{1}, \ldots, i_{k-1}, i_{k+1}, \ldots, i_{d}\right)=M\left(i_{1}, \ldots, i_{k-1}, x, i_{k+1}, \ldots, i_{d}\right)$ for all $0 \leq i_{j} \leq$ $m_{j}, j \in[1 . . d] \backslash\{k\}$. Finally, for $k \in[1 . . d]$ we define $\# \operatorname{sub}_{k}(M)$ as the number of different subarrays with fixed $k^{\text {th }}$ dimension:

$$
\# \operatorname{sub}_{k}(M)=\left|\left\{M_{x}^{(k)} \mid x \in\left[m_{k}\right]\right\}\right| .
$$

We call $M \in \mathcal{M}\left(m_{1}, \ldots, m_{d}\right)$ a Hankel array, if $M\left(i_{1}, \ldots, i_{d}\right)=M\left(j_{1}, \ldots, j_{d}\right)$ for every pair $\left(i_{1}, \ldots, i_{d}\right),\left(j_{1}, \ldots, j_{d}\right) \in\left[m_{1}\right] \times \ldots \times\left[m_{d}\right]$ with $i_{1}+\ldots+i_{d}=$ $j_{1}+\ldots+j_{d}$. For a Hankel array $M \in \mathcal{M}\left(m_{1}, \ldots, m_{d}\right)$, let $f_{M}:\left[\sum_{i=1}^{d} m_{i}\right] \rightarrow \Sigma$ be defined by $f_{M}(x)=M\left(x_{1}, \ldots, x_{d}\right)$, if $x=x_{1}+\ldots+x_{d}$. Note that $f_{M}$ is well-defined since $M$ is a Hankel array.

Lemma 8 For a function $f$ such that the corresponding communication array $M$ is a Hankel array, we have $r_{f_{M}}\left(m_{k}\right)=\# \operatorname{sub}_{k}(M)$ for every $k \in[1 . . d]$.

Proof: Since the value of the function $f$ depends on the sum of its variables, it is sufficient to show the claim for $k=1$.

Assume that for $x_{1}, x_{1}^{\prime} \in\left[m_{1}\right]$ the corresponding subarrays $M_{x_{1}}^{(1)}$ and $M_{x_{1}^{\prime}}^{(1)}$ are different. Then there exists $x_{2} \in\left[m_{2}\right], \ldots, x_{d} \in\left[m_{d}\right]$ such that $M\left(x_{1}, x_{2}, \ldots, x_{d}\right) \neq$ $M\left(x_{1}^{\prime}, x_{2}, \ldots, x_{d}\right)$ and therefore $f_{M}\left(x_{1}+x_{2}+\ldots+x_{d}\right) \neq f_{M}\left(x_{1}^{\prime}+x_{2}+\ldots+x_{d}\right)$ and $f_{m_{1}}\left(x_{1}, x_{2}+\ldots+x_{d}\right) \neq f_{m_{1}}\left(x_{1}^{\prime}, x_{2}+\ldots+x_{d}\right)$. Hence if two subarrays $M_{x_{1}}^{(1)}$ and $M_{x_{1}^{\prime}}^{(1)}$ are different, then also the rows $x_{1}$ and $x_{1}^{\prime}$ in $M_{f_{m_{1}}}$ are different, too. This implies $r_{f_{M}}\left(m_{1}\right) \geq \# \operatorname{sub}_{1}(M)$.

Analogously, let us assume that for $x_{1}, x_{1}^{\prime} \in\left[m_{1}\right]$ the rows in $M_{f_{m_{1}}}$ are different. Then there exists $y \in\left[\sum_{i \in[2 . . d]} m_{i}\right]$ such that $f_{m_{1}}\left(x_{1}, y\right) \neq f_{m_{1}}\left(x_{1}^{\prime}, y\right)$. Choosing $x_{2} \in\left[m_{2}\right], \ldots, x_{d} \in\left[m_{d}\right]$ such that $y=x_{2}+\ldots+x_{d}$ we get

$$
\begin{aligned}
& M\left(x_{1}, x_{2}, \ldots, x_{d}\right)=f_{M}\left(x_{1}+x_{2}+\ldots+x_{d}\right)=f_{m_{1}}\left(x_{1}, y\right) \\
\neq \quad & f_{m_{1}}\left(x_{1}^{\prime}, y\right)=f_{M}\left(x_{1}^{\prime}+x_{2}+\ldots+x_{d}\right)=M\left(x_{1}^{\prime}, x_{2}, \ldots, x_{d}\right) .
\end{aligned}
$$


Hence if rows $x_{1}$ and $x_{1}^{\prime}$ in $M_{f_{m_{1}}}$ are different then also the two subarrays $M_{x_{1}}^{(1)}$ and $M_{x_{1}^{\prime}}^{(1)}$ are different. This implies $r_{f_{M}}\left(m_{1}\right) \leq \# \operatorname{sub}_{1}(M)$.

To study communication complexity issues for multi-party computations, we first consider the following natural extension of the one-way communication model to multiple parties. Let $P_{1}, \ldots, P_{d}$ be connected by a directed chain specified by a permutation $\pi:[1 . . d] \rightarrow[1 . . d]$, i.e. $P_{\pi(i)}$ can only send messages to $P_{\pi(i+1)}$ for $i \in[d-1]$. Let $S^{\pi}(f)$ be the size of an optimal protocol on a chain specified by $\pi$ computing $f$. More precisely, $S^{\pi}(f)$ is the number of possible communication sequences on the network in an optimal protocol for $f$ (of that specific kind).

We will now present a protocol of minimal size for a fixed chain network and functions $f$ such that $M_{f}$ is a Hankel array. During the computation the parties use the Hankel arrays $M_{i}$ defined by

$$
M_{i}\left(y_{i}, \ldots, y_{d}\right)=M_{f}\left(z_{1}, \ldots, z_{d}\right)
$$

where $y_{i}=\sum_{j=1}^{i} z_{\pi(j)}$ and $y_{j}=z_{\pi(j)}$ for all $j \in[i+1 . . d]$. Furthermore, let $\Gamma_{i}\left(y_{i}\right)$ be the minimum value $z$ such that $\left(M_{i}\right)_{z}^{(1)}=\left(M_{i}\right)_{y_{i}}^{(1)}$. The protocol works as follows:

1. $P_{\pi(1)} \operatorname{sends} \gamma_{1}=\Gamma_{1}\left(x_{\pi(1)}\right)$ to $P_{\pi(2)}$.

2. For $i \in[2 . . d-1], P_{\pi(i)}$ receives $\gamma_{i-1}$ from $P_{\pi(i-1)}$ and sends $\gamma_{i}=\Gamma_{i}\left(x_{\pi(i)}+\gamma_{i-1}\right)$ to $P_{\pi(i+1)}$.

3. $P_{\pi(d)}$ receives $\gamma_{d-1}$ from $P_{\pi(d-1)}$. Then $M_{d}\left(\gamma_{d-1}+x_{\pi(d)}\right)$ is the result of the function.

Theorem 8 For a function $f$ such that $M_{f} \in \mathcal{M}\left(m_{1}, \ldots, m_{d}\right)$ is a Hankel array, the size of the protocol presented above is minimal.

Before giving a proof of the theorem we provide some auxiliary notions. If a protocol $\mathcal{P}$ runs on a chain $P_{\pi(1)}, \ldots, P_{\pi(n)}$, then we call $\mathcal{P} \pi$-ordered. For a given $\pi$-ordered protocol $\mathcal{P}$ let $S(\mathcal{P})$ be the number of different communication sequences of $\mathcal{P}$ and let $S(\mathcal{P}, r)$ be the number of different communication sequences of $\mathcal{P}$ on inputs $z_{1} \in\left[m_{1}\right], \ldots, z_{d} \in\left[m_{d}\right]$ with $f\left(z_{1}, \ldots, z_{d}\right)=r$. Finally, for a function $f$ : $\left[m_{1}\right] \times \ldots \times\left[m_{d}\right] \rightarrow \Sigma$ and a value $r \in \Sigma$ let

$$
S^{\pi}(f, r)=\min _{\mathcal{P} \text { computes } f \text { and is } \pi \text {-ordered }} S(\mathcal{P}, r) .
$$

The theorem above follows from the following lemma:

Lemma 9 Let $f$ be a function such that $M_{f} \in \mathcal{M}\left(m_{1}, \ldots, m_{d}\right)$ is a Hankel array. Then a $\pi$-ordered protocol $\mathcal{P}$ is optimal according to $S(\mathcal{P})$ and $S(\mathcal{P}, r)$ for every $r \in \Sigma$ iff for each $i \in[1 . . d-1]$ the message sent by the party $P_{\pi(i)}$ to $P_{\pi(i+1)}$ only depends on the subfunction of $f$ obtained by fixing the inputs of $P_{\pi(1)}, \ldots, P_{\pi(i)}$ and on the message received by $P_{\pi(i)}$. 
Proof: Let us first assume that there exists a party $P_{\pi(i)}$ with $i \in[1 . . d-1]$ and two inputs $x_{\pi(1)}, \ldots, x_{\pi(i)}$ and $y_{\pi(1)}, \ldots, y_{\pi(i)}$ such that (1) they specify two different subfunctions $f_{x}, f_{y}$ of $f$ and (2) $P_{\pi(i)}$ sends the same message to $P_{\pi(i+1)}$ for both inputs. Since $f_{x}, f_{y}$ are different functions, there exists an input $z_{\pi(i+1)}, \ldots, z_{\pi(d)}$ for the parties $P_{\pi(i+1)}, \ldots, P_{\pi(d)}$ such that $f_{x}$ and $f_{y}$ result in different values. Since the parties $P_{\pi(i+1)}, \ldots, P_{\pi(d)}$ cannot distinguish between both inputs, the protocol computes an incorrect value for at least one input. On the other hand, we do not increase $S(\mathcal{P})$ and $S(\mathcal{P}, r)$ if $P_{\pi(i)}$ adds some information about the received message to the message it is going to send.

Let us now assume that there exists $i \in[1 . . d-1]$ such that for two different partial inputs $x_{\pi(1)}, \ldots, x_{\pi(i)}$ and $y_{\pi(1)}, \ldots, y_{\pi(i)}$ that specify the same subfunction $f^{\prime}$ of $f$, the party $P_{\pi(i)}$ receives the same message but sends two different messages to $P_{\pi(i+1)}$. Let $X$ be the set of all inputs where the values of $P_{\pi(1)}, \ldots, P_{\pi(i)}$ are given by $x_{\pi(1)}, \ldots, x_{\pi(i)}$. For $x \in X$ let $Y_{x}$ denote the set of all inputs for which $P_{\pi(i)}$ receives the same message from its predecessor as for the input $x$, the input of $P_{\pi(i)}$ is given by $z_{\pi(i)}$ and the input of $P_{\pi(j)}$ is given by $x_{\pi(j)}$ for all $j \in[i+1 . . d]$.

Note that $f(x)=f(y)$ for every $x \in X$ and every $y \in Y_{x}$. Hence we do not increase the size of the protocol if $P_{\pi(i)}$ sends on both inputs the same messages to $P_{\pi(i+1)}$. Moreover, if every party only sends to its successor a unique message for each $y \in Y_{x}$ we reduce total size as well as the size of $S(\mathcal{P}, f(x))$.

Note that the communication size $S^{\pi}$ may depend on the order $\pi$ of the parties on the chain. We call a permutation $\pi:[1 . . d] \rightarrow[1 . . d]$ with $m_{\pi(i)} \leq m_{\pi(i+1)}$ for all $i \in[1 . . d-1]$ a grading of $m_{1}, \ldots, m_{d}$. Consequently, for a grading $\pi$ of $m_{1}, \ldots, m_{d}$, the ordering is optimal with respect to the communication size.

Theorem 9 Let $f$ be a function such that $M_{f} \in \mathcal{M}\left(m_{1}, \ldots, m_{d}\right)$ is a Hankel array, and let $\pi$ be a grading of $m_{1}, \ldots, m_{d}$. Then for every permutation $\pi^{\prime}:[1 . . d] \rightarrow[1 . . d]$ $S^{\pi}(f) \leq S^{\pi^{\prime}}(f)$.

To prove the theorem above, we present a more general result:

Lemma 10 Let $f$ be a function such that $M_{f} \in \mathcal{M}\left(m_{1}, \ldots, m_{d}\right)$ is a Hankel array, $\pi$ be a grading of $m_{1}, \ldots, m_{d}$, and $\mathcal{P}$ be a $\pi$-ordered protocol for $f$ that is optimal with respect to protocol size. Then for every permutation $\pi^{\prime}:[1 . . d] \rightarrow[1 . . d]$

$$
S(\mathcal{P}) \leq S^{\pi^{\prime}}(f) \text { and } \forall r \in \Sigma: S(\mathcal{P}, r) \leq S^{\pi^{\prime}}(f, r)
$$

Proof: Within this proof let $M=M_{f}$ for short.

The proof follows by induction on $d$. For $d=2$ each protocol corresponds to a one-way two-party protocol. Hence from Theorem 4 we can conclude that for every grading $\pi$ of $m_{1}, m_{2}$ a $\pi$-ordered protocol $\mathcal{P}$ is optimal with respect to protocol size if $P_{1}$ only specifies the type of the row of $M$ which is determined by its input.

The proof of the second condition of the lemma above is more complicated. For a permutation $\pi^{\prime}:\{1,2\} \rightarrow\{1,2\}$ let $\mathcal{P}^{\prime}$ be an arbitrary $\pi^{\prime}$-ordered protocol computing 
$f$ on the two-party chain. Then each message sent from $P_{\pi^{\prime}(1)}$ to $P_{\pi^{\prime}(2)}$ specifies a subset of subarrays $M_{x}^{\pi^{\prime}(1)}$ with $x \in\left[m_{\pi^{\prime}(1)}\right]$ of $M$. Hence the size $S\left(\mathcal{P}^{\prime}, r\right)$ is at least as large as the number of different subarrays $M_{x}^{\pi^{\prime}(1)}$ which contain $r$. Hence a $\pi^{\prime}$ ordered protocol $\mathcal{P}^{\prime \prime}$ that is optimal with respect to $S\left(\mathcal{P}^{\prime \prime}, r\right)$ uses only one message for every type of subarray (see Lemma 9 ). This implies

$$
S(\mathcal{P})=S^{\pi^{\prime}}(f) \quad \text { and } \quad \forall r \in \Sigma: S(\mathcal{P}, r)=S^{\pi^{\prime}}(f, r)
$$

for every grading $\pi^{\prime}$ of $m_{1}, m_{2}$.

Let us now assume that $\pi^{\prime}$ is not a grading of $m_{1}, m_{2}$ and $\mathcal{P}^{\prime}$ is an $\pi^{\prime}$-ordered protocol that is optimal with respect to $S\left(\mathcal{P}^{\prime}, r\right)$. For easier notation assume that $\pi^{\prime}(1)=2$ and $\pi^{\prime}(2)=1$. Hence for a grading $\pi$ of $m_{1}, m_{2}$ we have $\pi(1)=1$ and $\pi(2)=2$. Let $\mathcal{P}$ be an optimal $\pi$-ordered protocol. We consider the following cases:

1. If for every $x_{2} \in\left[m_{2}\right], r$ appears in $M_{x_{2}}^{(2)}$, then $r$ also appears in every subarray $M_{x_{1}}^{(1)}$ with $x_{1} \in\left[m_{1}\right]$. Hence $S^{\pi^{\prime \prime}}(f, r)=S^{\pi^{\prime \prime}}(f)$ for every permutation $\pi^{\prime \prime}$. The claim follows directly from Theorem 4 .

2. There exists no $x_{2} \in\left[m_{2}\right]$ such that $r$ appears in $M_{x_{2}}^{(2)}$. Then $f\left(x_{1}, x_{2}\right) \neq r$ for every input $x_{1} \in\left[m_{1}\right], x_{2} \in\left[m_{2}\right]$. The claim follows directly.

3. There exists $x_{2}, x_{2}^{\prime} \in\left[m_{2}\right]$ such that $r$ does appear in $M_{x_{2}}^{(2)}$ but not in $M_{x_{2}^{\prime}}^{(2)}$.

(a) $r$ does not appear in $M_{0}^{(2)}$. Then choose $x_{2}^{\prime} \in\left[m_{2}\right]$ to be maximal such that $r$ does not appear in $M_{x}^{(2)}$ for every $x \in\left[x_{2}^{\prime}\right]$. Hence $r$ appears in $M_{x^{\prime}}^{(2)}$ for every $x_{2}^{\prime}+1 \leq x^{\prime} \leq \min \left\{m_{2}, x_{2}^{\prime}+m_{1}+1\right\}$. This case is illustrated in Figure 2.

If $x_{2}^{\prime}+m_{1}+1 \leq m_{2}$, then there are at least $m_{1}+1$ different subarrays $M_{x^{\prime}}^{(2)}$ which contain $r$, e.g. the subarrays $M_{x_{2}^{\prime}+1}^{(2)}$ to $M_{x_{2}^{\prime}+m_{1}+1}^{(2)}$. Since the number of different communication strings of a $\pi$-ordered protocol $\mathcal{P}$ is bounded by $m_{1}+1$, the claim follows directly.

If $x_{2}^{\prime}+m_{1}+1>m_{2}$, then the number of different subarrays $M_{x^{\prime}}^{(2)}$ which contain $r$ is $m_{2}-x_{2}^{\prime}$. Since $M$ is a Hankel array, the number of different subarrays $M_{x^{\prime \prime}}^{(1)}$ with $x^{\prime \prime} \in\left[m_{1}\right]$ which contain $r$ is $m_{2}-x_{2}^{\prime}$, too - the claim follows directly.

(b) $r$ does not appear in $M_{m_{2}}^{(2)}$. This case is symmetric to case 3.a and follows analogously.

(c) There exists $z_{2}, z_{2}^{\prime} \in\left[m_{2}\right]$ with $z_{2}<x_{2}^{\prime}<z_{2}^{\prime}$ such that $r$ appears in $M_{z_{2}}^{(2)}$ and $M_{z_{2}^{\prime}}^{(2)}$. Then we choose $z_{2}$ maximal and $z_{2}^{\prime}$ minimal such that the condition above is fulfilled. Note that $r$ does not appear in the subarrays 


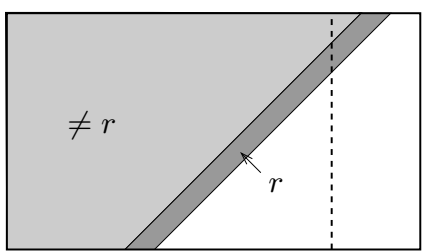

Figure 2: An illustration of Case 3.a.

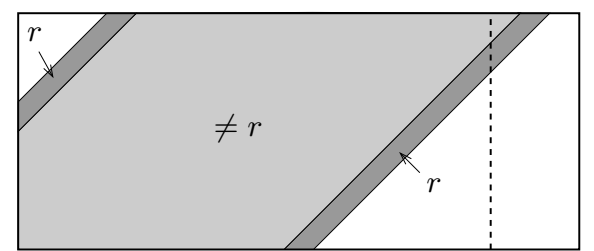

Figure 3: An illustration of Case 3.c.

$M_{z_{2}+1}^{(2)}$ to $M_{z_{2}^{\prime}-1}^{(2)}$. Since $M$ is a Hankel array for every $\ell \in\left[m_{1}-1\right]$, the subarrays $M_{x}^{(2)}$ with

$$
x \in\left\{\max \left\{0, z_{2}-\ell\right\}, \ldots, z_{2}\right\} \cup\left\{z_{2}^{\prime}, \ldots, \min \left\{m_{2}, z_{2}^{\prime}+m_{1}-\ell-1\right\}\right\}
$$

are pairwise different and contain $r$. This implies that the number of different subarrays $M_{x}^{(2)}$ with $x \in\left[m_{2}\right]$ containing $r$ is at least $\min \left\{m_{1}+\right.$ $\left.1, m_{2}-z_{2}^{\prime}+z_{2}+2\right\}$. Hence for $m_{1}+1 \leq m_{2}-z_{2}^{\prime}+z_{2}+2$ the claim follows directly.

Let us now assume that $m_{1}+1>m_{2}-z_{2}^{\prime}+z_{2}+2$. Since $M$ is a Hankel array, $r$ appears only in $M_{x}^{(1)}$ if $x \in\left\{0, \ldots, z_{2}\right\} \cup\left\{m_{1}-\left(m_{2}-z_{2}^{\prime}\right), \ldots, m_{1}\right\}$. Hence the number of subarrays $M_{x}^{(1)}$ with $x \in\left[m_{1}\right]$ containing $r$ is $m_{2}-$ $z_{2}^{\prime}+z_{2}+2$.

Let us now investigate the case that $d>2$, that means that the chain contains more than two parties $P_{1}, \ldots, P_{d}$. We will show that for every grading $\pi$ of $m_{1}, \ldots, m_{d}$, there exists a $\pi$-ordered protocol $\mathcal{P}$ such that for every permutation $\pi_{s}:[1 . . d] \rightarrow[1 . . d]$ and for every $\pi_{s}$-ordered protocol $\mathcal{P}_{s}$ it holds:

$$
S(\mathcal{P}) \leq S\left(\mathcal{P}_{s}\right) \quad \text { and } \quad \forall r \in \Sigma: S(\mathcal{P}, r) \leq S\left(\mathcal{P}_{s}, r\right)
$$

Note that for two permutations $\pi_{1}, \pi_{2}$ which are equivalent in the sense that for every $i \in[1 . . d] m_{\pi_{1}(i)}=m_{\pi_{2}(i)}$, we can simulate any $\pi_{1}$-ordered protocol on a chain that is given by the permutation $\pi_{2}$ without increasing the size of the protocol. Hence it is sufficient to show that (3) holds for some grading $\pi$ of $m_{1}, \ldots, m_{d}$.

For $i \in[1 . . d]$ and $x \in\left[m_{i}\right]$ let $f_{x}^{(i)}$ denote the integer function corresponding to $M_{x}^{(i)}$. Furthermore, let $\pi_{x}^{(i)}$ be a grading of $m_{1}, \ldots, m_{i-1}, m_{i+1}, \ldots, m_{d}$, and let $\mathcal{P}_{x}^{(i)}$ be a $\pi_{x}^{(i)}$-ordered protocol for $f_{x}^{(i)}$ that fulfills the conditions of Lemma 10.

Since for every input $x \in\left[m_{\pi_{s}(1)}\right] \quad M_{x}^{\left(\pi_{s}(1)\right)}$ is a Hankel array, we can apply the induction hypothesis to prove the existence of an optimal $\pi_{x}^{\left(\pi_{s}(1)\right)}$-ordered protocol $\mathcal{P}_{x}^{\left(\pi_{s}(1)\right)}$ for $f_{x}^{\left(\pi_{s}(1)\right)}$ such that for every permutation $\pi^{\prime}:[1 . . d-1] \rightarrow[1 . . d-1]$ and every $r \in \Sigma$

$$
S\left(\mathcal{P}_{x}^{\left(\pi_{s}(1)\right)}\right) \leq S^{\pi^{\prime}}\left(f_{x}^{\left(\pi_{s}(1)\right)}\right) \quad \text { and } \quad S\left(\mathcal{P}_{x}^{\left(\pi_{s}(1)\right)}, r\right) \leq S^{\pi^{\prime}}\left(f_{x}^{\left(\pi_{s}(1)\right)}, r\right) .
$$


This implies that for every permutation $\pi^{\prime \prime}:[1 . . d] \rightarrow[1 . . d]$ with $\pi^{\prime \prime}(1)=\pi_{s}(1)$ there exists a protocol $\mathcal{P}_{h}$ on the chain that starts with $P_{\pi_{s}(1)}$ and where the remaining parties are $\pi_{x}^{\left(\pi_{s}(1)\right)}$-ordered such that

$$
S\left(\mathcal{P}_{h}\right)=\sum_{M_{x}^{\left(\pi_{s}(1)\right)}} S\left(\mathcal{P}_{x}^{\left(\pi_{s}(1)\right)}\right) \leq \sum_{M_{x}^{\left(\pi_{s}(1)\right)}} S^{\pi^{\prime \prime \prime}}\left(f_{x}^{\left(\pi_{s}(1)\right)}\right)=S^{\pi^{\prime \prime}}(f)
$$

and

$$
S\left(\mathcal{P}_{h}, r\right)=\sum_{M_{x}^{\left(\pi_{s}(1)\right)}} S\left(\mathcal{P}_{x}^{\left(\pi_{s}(1)\right)}, r\right) \leq \sum_{M_{x}^{\left(\pi_{s}(1)\right)}} S^{\pi^{\prime \prime \prime}}\left(f_{x}^{\left(\pi_{s}(1)\right)}, r\right)=S^{\pi^{\prime \prime}}(f, r)
$$

where $\pi^{\prime \prime \prime}$ denotes the permutation that corresponds to $\pi^{\prime \prime}$ without $\pi(1)$. The protocol $\mathcal{P}_{h}$ can be constructed as follows: The first party $P_{\pi_{s}(1)}$ computes the type of the subarray $M_{x}^{\left(\pi_{s}(1)\right)}$ with $x \in\left[m_{\pi_{s}(1)}\right]$ that is given by its input $x_{\pi_{s}(1)}$. The remaining parties simulate the protocol $\mathcal{P}_{x}^{\left(\pi_{s}(1)\right)}$. Note that if $\pi_{h}$ is a grading of $m_{1}, \ldots, m_{d}$ then the claim follows directly.

Let us focus now on the case that $\pi_{h}$ is not a grading of $m_{1}, \ldots, m_{d}$. Since $\pi_{h}(i)$ is a grading of $m_{\pi_{h}(2)}, \ldots, m_{\pi_{h}(d)}$, the value $m_{\pi_{h}(2)}$ is minimal for all values $m_{1}, \ldots, m_{d}$.

We proceed in two steps:

1. In the first step, we will investigate permutations $\tilde{\pi}_{h}$ that are similar to $\pi_{h}$ except for the first two values, i.e. $m_{\tilde{\pi}_{h}(1)}=m_{\pi_{h}(2)}$ and $m_{\tilde{\pi}_{h}(2)}=m_{\pi_{h}(1)}$.

2. In the second step, we will investigate permutations $\pi_{h}^{\prime}$ with $\pi_{h}^{\prime}(1)=\tilde{\pi}_{h}(1)$.

For any $x_{1} \in\left[m_{\pi_{h}(1)}\right]$ and $x_{2} \in\left[m_{\pi_{h}(2)}\right]$ define $f_{x_{1}, x_{2}}$ as the subfunction of $f$ where we assign to the $\pi_{h}(1)$-th and $\pi_{h}(2)$-th variable of $f$ the values $x_{1}$ and $x_{2}$, respectively. Furthermore, let $M_{x_{1}, x_{2}}$ denote the communication array of $f_{x_{1}, x_{2}}$. Note that $M_{x_{1}, x_{2}}$ is a Hankel array, too.

Let us now divide $\mathcal{P}_{h}$ into two parts. The first part $\mathcal{P}_{h}^{1}$ consists of the strategies for the first two parties $P_{\pi_{h}(1)}$ and $P_{\pi_{h}(2)}$. If $\mathcal{P}_{h}$ is optimal according to its size $S\left(\mathcal{P}_{h}\right)$ and $S\left(\mathcal{P}_{h}, r\right)$ with respect to all $\pi_{h}$-ordered protocols, we can assume that $P_{\pi_{h}(2)}$ only sends the type of the subarray $M_{x_{1}, x_{2}}$ to $P_{\pi_{h}(3)}$.

The second part $\mathcal{P}_{h}^{2}\left(M_{x_{1}, x_{2}}\right)$ of $\mathcal{P}_{h}$ consists of the strategies for the remaining $d-2$ parties $P_{\pi_{h}(3)}$ to $P_{\pi_{h}(d)}$ where the input of the first two parties is given by $x_{1}$ and $x_{2}$, respectively. Let $\Sigma_{M}$ be the set of all subarrays $M_{x_{1}, x_{2}}$, then we have

$$
S\left(\mathcal{P}_{h}\right)=\sum_{M_{x_{1}, x_{2}} \in \Sigma_{M}} S\left(\mathcal{P}_{h}^{1}, M_{x_{1}, x_{2}}\right) \cdot S\left(\mathcal{P}_{h}^{2}\left(M_{x_{1}, x_{2}}\right)\right)
$$

and for all $r$ in the range of $f$ we have

$$
S\left(\mathcal{P}_{h}, r\right)=\sum_{M_{x_{1}, x_{2}} \in \Sigma_{M}} S\left(\mathcal{P}_{h}^{1}, M_{x_{1}, x_{2}}\right) \cdot S\left(\mathcal{P}_{h}^{2}\left(M_{x_{1}, x_{2}}\right), r\right)
$$


Let $g:\left[m_{\pi_{h}(1)}\right] \times\left[m_{\pi_{h}(2)}\right] \rightarrow \Sigma_{M}$ be the function that is computed by the first two parties in the chain. Note that the communication array $M_{g}$ of $g$ is a Hankel array again.

Let $\tilde{\pi}_{h}$ be a permutation with $\tilde{\pi}_{h}(1)=\pi_{h}(2), \tilde{\pi}_{h}(2)=\pi_{h}(1)$, and $\tilde{\pi}_{h}(i)=\pi_{h}(i)$ for all $i \in[3 . . d]$. We will now investigate the $\tilde{\pi}_{h}$-ordered protocol $\tilde{\mathcal{P}}_{h}$ that is defined as follows: $\tilde{\mathcal{P}}_{h}$ runs an optimal strategy to compute $g$ on the first two parties and simulates $\mathcal{P}_{h}$ on the remaining parties. Analogously to the partition of $\mathcal{P}_{h}$, we partition $\tilde{\mathcal{P}}_{h}$ into two parts $\tilde{\mathcal{P}}_{h}^{1}$ and $\tilde{\mathcal{P}}_{h}^{2}$. From the base case with $d=2$ we can conclude that there exists such a subprotocol $\tilde{\mathcal{P}}_{h}^{1}$ with

$$
\begin{aligned}
S\left(\tilde{\mathcal{P}}_{h}\right) & =\sum_{M_{x_{1}, x_{2}} \in \Sigma_{M}} S\left(\tilde{\mathcal{P}}_{h}^{1}, M_{x_{1}, x_{2}}\right) \cdot S\left(\tilde{\mathcal{P}}_{h}^{2}\left(M_{x_{1}, x_{2}}\right)\right) \\
& \leq \sum_{M_{x_{1}, x_{2}} \in \Sigma_{M}} S\left(\mathcal{P}_{h}^{1}, M_{x_{1}, x_{2}}\right) \cdot S\left(\tilde{\mathcal{P}}_{h}^{2}\left(M_{x_{1}, x_{2}}\right)\right)=S\left(\mathcal{P}_{h}\right)
\end{aligned}
$$

and

$$
\begin{aligned}
S\left(\tilde{\mathcal{P}}_{h}, r\right) & =\sum_{M_{x_{1}, x_{2}} \in \Sigma_{M}} S\left(\tilde{\mathcal{P}}_{h}^{1}, M_{x_{1}, x_{2}}\right) \cdot S\left(\tilde{\mathcal{P}}_{h}^{2}\left(M_{x_{1}, x_{2}}\right), r\right) \\
& \leq \sum_{M_{x_{1}, x_{2}} \in \Sigma_{M}} S\left(\mathcal{P}_{h}^{1}, M_{x_{1}, x_{2}}\right) \cdot S\left(\tilde{\mathcal{P}}_{h}^{2}\left(M_{x_{1}, x_{2}}\right), r\right)=S\left(\mathcal{P}_{h}, r\right)
\end{aligned}
$$

for all $r$ in the range of $f$.

Analogously to the construction of $\mathcal{P}_{h}$ we can now apply a transformation on $\tilde{\mathcal{P}}_{h}$ to get a $\pi_{h}^{\prime}$-ordered protocol $\mathcal{P}_{h}^{\prime}$ for a permutation $\pi_{h}^{\prime}(1)=\tilde{\pi}_{h}(1)$ and $m_{\pi_{h}^{\prime}(i)} \leq m_{\pi_{h}^{\prime}(i+1)}$ for all $i \in[2 . . d-1]$ such that $\mathcal{P}_{h}^{\prime}$ fulfills the following inequalities:

$$
S\left(\mathcal{P}_{h}^{\prime}\right) \leq S\left(\tilde{\mathcal{P}}_{h}\right) \leq S\left(\mathcal{P}_{h}\right) \leq S^{\pi_{s}}(f) \leq S\left(\mathcal{P}_{s}\right)
$$

and for all $r$ in the range of $f$

$$
S\left(\mathcal{P}_{h}^{\prime}, r\right) \leq S\left(\tilde{\mathcal{P}}_{h}, r\right) \leq S\left(\mathcal{P}_{h}, r\right) \leq S^{\pi_{s}}(f, r) \leq S\left(\mathcal{P}_{s}, r\right)
$$

for every $\pi_{s}$-ordered protocol $\mathcal{P}_{s}$.

Since $m_{\tilde{\pi}_{h}(1)}$ is minimal for all values $m_{1}, \ldots, m_{d}$ and the values $m_{\pi_{h}^{\prime}(i)}$ for $i \in$ $[2 . . d]$ are ordered according to their size, we can conclude that $\pi_{h}^{\prime}(i)$ is a grading of $m_{1}, \ldots, m_{d}$.

A second generalization of the two-party model is the simultaneous communication complexity (denoted by $C^{\|}$), where all parties can simultaneously write in a single round on a blackboard. This means that the messages sent by each party do not depend on the messages sent by the other parties. After finishing the communication round, each party has to be able to compute the result of the function (see e.g. [9]). For two-party communication it is well-known that

$$
C^{\|}(f)=C^{A \rightarrow B}(f)+C^{B \rightarrow A}(f)=\left\lceil\log S^{A \rightarrow B}(f)\right\rceil+\left\lceil\log S^{B \rightarrow A}(f)\right\rceil .
$$


Similarly, for the $d$-party case we have

$$
C^{\|}(f)=\sum_{i \in[1 . . d]}\left\lceil\log \# \operatorname{sub}_{i}\left(M_{f}\right)\right\rceil .
$$

Hence if $M_{f}$ is a Hankel array and if for some $k \in[1 . . d]$ we have \#sub ${ }_{k}\left(M_{f}\right) \leq$ $\min _{i \in[1 . . d]} m_{i}$, then by Lemmas 7 and 8

$$
C^{\|}(f)=d \cdot\left\lceil\log \Delta\left(f_{M_{f}}\right)\right\rceil
$$

As a third generalization, we consider the case that in each round some party can write a message on a blackboard. The message and its sender may depend on messages that have been published on the board in previous rounds. We restrict the communication such that each party (except for the last one) publishes exactly one message on the blackboard, and in each round exactly one message is published. After finishing the communication rounds, at least one party has to be able to compute the result of the function. Let $S^{\square}$ be the corresponding size of an optimal protocol. Note that this model generalizes both of the previous models.

Theorem 10 Let $f$ be a function such that $M_{f} \in \mathcal{M}\left(m_{1}, \ldots, m_{d}\right)$ is a Hankel array and let $\pi$ be a grading of $m_{1}, \ldots, m_{d}$. Then $S^{\pi}(f)=S^{\square}(f)$.

Proof: The proof follows by complete induction on the number of parties $d$. For $d=2$ the claim follows directly from the standard one-way two-party scenario.

For $d>2$ let us first note that the first party that writes a message to the blackboard has to be determined by the protocol independently of the concrete input. Let $P_{k}$ be the party that writes its message first. The second party that writes a message on the blackboard may depend on the type of $M_{x_{k}}^{(k)}$ where $z_{k} \in\left[m_{k}\right]$ is the input of $P_{k}$. Let $f_{x_{k}}^{(k)}$ describe the function with communication array $M_{x_{k}}^{(k)}$. Since $M_{x_{k}}^{(k)}$ is a Hankel array too, we can apply the induction hypothesis to the computation of $f_{x_{k}}^{(k)}$. Note that $M_{x_{k}}^{(k)} \in \mathcal{M}\left(m_{1}, \ldots, m_{k-1}, m_{k+1}, \ldots, m_{d}\right)$. Hence for a grading $\pi_{k}:[1 . . d-1] \rightarrow$ $[1 . . d-1]$ of $m_{1}, \ldots, m_{d-1}$ we have:

$$
S^{\pi_{k}}\left(f_{x_{k}}^{(k)}\right)=S^{\square}\left(f_{x_{k}}^{(k)}\right) .
$$

Since the first party that writes a message on the blackboard is chosen independently of the concrete input, this equation implies

$$
S^{\pi^{\prime}}(f)=\sum_{M_{z_{k}}^{(k)}} S^{\pi_{k}}\left(f_{z_{k}}^{(k)}\right)=\sum_{M_{z_{i}}^{(i)}} S^{\square}\left(f_{z_{i}}^{(i)}\right)=S^{\square}(f)
$$

where $\pi^{\prime}(1)=k$ and for $i \in[1 . . d-1] \pi^{\prime}(i+1)=\pi_{k}(i)$. By Theorem 9 we get for a grading $\pi:[1 . . d] \rightarrow[1 . . d]$ of $m_{1}, \ldots, m_{d}$ :

$$
S^{\pi}(f) \leq S^{\pi^{\prime}}(f)=S^{\square}(f) .
$$


On the other hand, we can always simulate a protocol which works on a chain by a protocol that uses a blackboard without increasing the size of the protocol. Hence

$$
S^{\square}(f) \leq S^{\pi}(f) .
$$

The claim follows directly.

\section{Conclusions and Open Problems}

In this paper we have investigated one-way communication complexity of functions for which the corresponding communication matrices are Hankel matrices. We have established some structural properties of such matrices. As a direct application, we have obtained a complete solution to the problem of how the communication direction in deterministic one-way communication protocols affects the communication complexity of symmetric Boolean functions. One possible direction of future research is to study other kinds of one-way communication such as nondeterministic and randomized for the class of symmetric functions.

Another interesting extension of the topic is to drop the restriction to one-way protocols and consider the deterministic two-way communication complexity of symmetric Boolean functions for both a bounded and an unbounded number of communication rounds. This particularly involves results about the computation of the rank of Hankel matrices. In addition, consequences for word combinatorics and OBDD theory may be of interest.

Acknowledgment: We would like to thank Ingo Wegener for his useful comment on the connection between one-way communication and OBDD theory.

\section{References}

[1] F. Ablayev, Lower bounds for one-way probabilistic communication complexity and their application to space complexity. Theoretical Comp. Sc., 157 (1996), 139-159.

[2] A. Condon, L. Hellerstein, S. Pottle, and A. Wigderson, On the power of finite automata with both nondeterministic and probabilistic states. SIAM J. Comput., 27 (1998), 739-762.

[3] P. Ďuriš, J. Hromkovič, J.D.P. Rolim, and G. Schnitger, On the power of Las Vegas for one-way communication complexity, finite automata, and polynomialtime computations. Proc. 14th STACS, Springer, 1997, 117-128.

[4] J. E. Hopcroft and J. D. Ullman, Formal Languages and Their Relation to Automata. Addison-Wesley, Reading, Massachusetts, 1969. 
[5] J. Hromkovič, Communication Complexity and Parallel Computing. Springer, 1997.

[6] I. S. Iohvidov, Hankel and Toeplitz Matrices and Forms. Birkh“auser, Boston, 1982

[7] H. Klauck, On quantum and probabilistic communication: Las Vegas and oneway protocols. Proc. 32nd STOC, 2000, 644-651.

[8] I. Kremer, N. Nisan, and D. Ron, On randomized one-round communication complexity. Computational Complexity, 8 (1999), 21-49.

[9] E. Kushilevitz and N. Nisan, Communication Complexity. Camb. Univ. Press, 1997.

[10] K. Mehlhorn and E. M. Schmidt, Las Vegas is better than determinism in VLSI and distributed computing. Proc. 14th STOC, 1982, 330-337.

[11] I. Newman and M. Szegedy, Public vs. private coin flips in one round communication games. Proc. 28th STOC, 1996, 561-570.

[12] C. Papadimitriou and M. Sipser, Communication complexity. J. Comput. System Sci., 28 (1984), 260-269.

[13] I. Wegener, Optimal decision trees and one-time-only branching programs for symmetric Boolean functions. Information and Control, 62 (1984), 129-143

[14] I. Wegener, The complexity of Boolean functions. Wiley-Teubner, 1987.

[15] I. Wegener, personal communication, April 2003.

[16] A. C. Yao, Some complexity questions related to distributive computing. Proc. 11th STOC, 1979, 209-213. 